\title{
Numerical Analysis of a Sling Support Arrangement for GRP Composite Pressure Vessels
}

\author{
D H Nash \& W M Banks
}

Department of Mechanical Engineering

University of Strathclyde

Glasgow, G1 1XJ

\begin{abstract}
A flexible sling support arrangement for horizontal glass reinforced plastic pressure vessels is examined using advanced finite element methods. A mathematical model is produced employing a suitable analysis capable of representing the non-linear behaviour of a sling supported GRP vessel. This system is used to examine the phenomena occurring at the interface between the vessel and the supporting belt. Each component is initially considered some distance apart and then brought together using three-dimensional contact surfaces. External loads are thereafter applied to the combined model. Although several numerical difficulties arise due to the difference in flexibility between the vessel shell and the sling support, these are overcome and the resulting vessel strains and contact interface pressures show good agreement with experimental work. The magnitudes of the strains at the location of the saddle horn are significantly reduced. Results of a parameter study are also presented which show the effect of the sling position together with the influence of the wrap-round angle and a number of recommendations are made with respect to design.
\end{abstract}

\section{Introduction}

Composite storage vessels can offer a distinct advantage over steel vessels, especially for the storage of hazardous liquids in the chemical process industry. Although many are aligned vertically with a skirt support, the horizontal vessel is particularly useful when there is a restriction on height and space. The most commonly used support system is the 'twin saddle arrangement' which is similar to that of Zick ${ }^{1}$ used for steel vessels. A second alternative is the 'longitudinal beam support' which forms an integral part of the structure and can run the full length of the vessel. The third alternative, the 'flexible sling system', provides support around the lower region of the cylindrical portion of the vessel. Although each of these three systems may be used in the support of GRP vessels, evidence is still required to enable a thorough failure assessment to be carried out, and to determine those applications and situations where their use is most appropriate.

Some guidance is provided in the British Standard BS4994 ${ }^{2}$ and also in the ASME Boiler and Pressure Vessel Code $^{3}$, Section X. However this information is only qualitative, with the exception of a reference in BS 4994 dealing with metallic pressure vessels. This indicates that 
in the case of a saddle support, the detailed analysis given in BS $5500^{4}$ may be used for GRP vessels providing caution is exercised with regard to the strain limitation. This somewhat simplistic, but safe, advice implies that it is relatively straightforward to determine the maximum strain from the analysis given in BS 5500. This treatment assumes the vessel is isotropic and does not address the multi-layered anisotropic case. In addition, the loose saddle support is recommended even though there are well known problems associated with this type of support.

Both the saddle support and beam support systems have been studied extensively and the work is thoroughly documented ${ }^{5,6}$. However, although some experimental studies have been carried out on the sling supported GRP vessel, there is no suitable analytical treatment as yet. This is due, in part, to the complex nature of the flexibility of the sling support and the interaction with the vessel shell. In this arrangement, a flexible sling fabricated from a strong material, say a Kevlar cloth, is used to carry the vessel rather than the vessel being supported by a more rigid steel saddle. Since the sling is highly flexible, it can change shape as the vessel deforms under load thus avoiding any rigid constraint or abrupt changes of section that occurs with rigid saddles. It is this rapid change of vessel shape that introduces highly localised bending stresses into the shell. This can lead to direct failure of the composite shell or reduce the life of the vessel by accelerating failure by fatigue for cyclically loaded systems.

Recent experimental and theoretical work undertaken ${ }^{6}$, based on thin shell theory and a Fourier series approach, has shown that the introduction of a flexible sling reduces the stresses arising at the traditional saddle horn location. Therefore this type of support should have potential application in GRP vessel design where displacements are larger than those found commonly in steel vessels and limiting the maximum vessel strain is often the major design criterion.

\section{Previous Work}

Work by Tooth et al. ${ }^{7,8,9,10}$ employs a shell analysis and Fourier series approach that evaluates the form of the contact interface pressure profile. This is undertaken by subdividing the contact region into a number of discrete areas around the circumference and across the saddle width. The flexibility of the support is introduced by the use of a flexibility matrix, generated by the application of unit loads on an equivalent model for the support. In the case of the saddle, this can be thought of as a tapered beam. Applying unit loads in the radial and tangential directions generates the flexibility matrix. Thereafter, the vessel shell is allowed to deform and by enforcing compatibility between the deformed surface and the imposed flexible 
support at the centre of the contact patch, the interface pressure can be evaluated and thereafter applied to the shell to solve for displacements and strains.

Although the method provides reasonable agreement with the limited tests presented in the literature, there are several drawbacks associated with this method which may be of importance when applied to the sling support case. Since the form of contact pressure between the shell and the support is unknown, and may change depending on the state of loading; this may become important. In addition, it can vary during the loading sequence when a sling is used, for example, when the vessel is empty, the self-weight induces one form of contact pressure profile. If the vessel is gradually filled, the contact pressure varies as the shell and slings adopt a new shape. If the filled vessel is subsequently pressurised, the shell stiffens and the sling adjusts its position. Therefore the form of contact pressure may vary and may not be uniform for all cases. The generation of a flexibility matrix for the support requires the sling to be modelled and the unit load displacement matrix to be evaluated. This is a reasonable approach for a steel saddle where the radial and tangential flexibility can be found using a beam analogy or by the use of finite elements. However, this is made somewhat more difficult when a sling is used, as the basic stiffness is very low and the sling behaves as a cloth material, therefore allowing the vessel to deform in such a manner that the problem may become nonlinear. The sling is able to carry a significant load when in tension but less in bending. However the greater the tension, the stiffer the sling.

Although Erzingatzian et $\mathrm{al}^{11}$ proposed an iterative method using finite element analysis to determine the reaction forces at the interface of a fluid loaded, filament wound, fibre reinforced plastic pipe resting on twin rigid saddles, there are some drawbacks with his approach. This is due to the procedure of repeatedly modifying fixed boundary conditions until full contact has been achieved. Although the method shows qualitative agreement with experimental measurements, a quantitative solution is still required. In addition, the problem of introducing the more flexible support remains ${ }^{12}$.

\section{Finite Element Approach using General Contact Surfaces}

A fully parametric finite element model has been developed by the present authors ${ }^{12}$ to address the problem of the difference in component flexibility. In addition, using this approach, the contact surface interface pressures are evaluated. This system has been used to examine the phenomena occurring at the interface between the vessel and supporting belt. 


\section{Contact Elements and Contact Stiffness}

In order to represent the contact between the flexible support and the stiffer shell, the vessel and belt are modelled as discrete components and 'general surface contact elements' were introduced to represent contact and sliding between the two mating surfaces in three dimensions. The contact elements have five nodes with three degrees of freedom at each node.

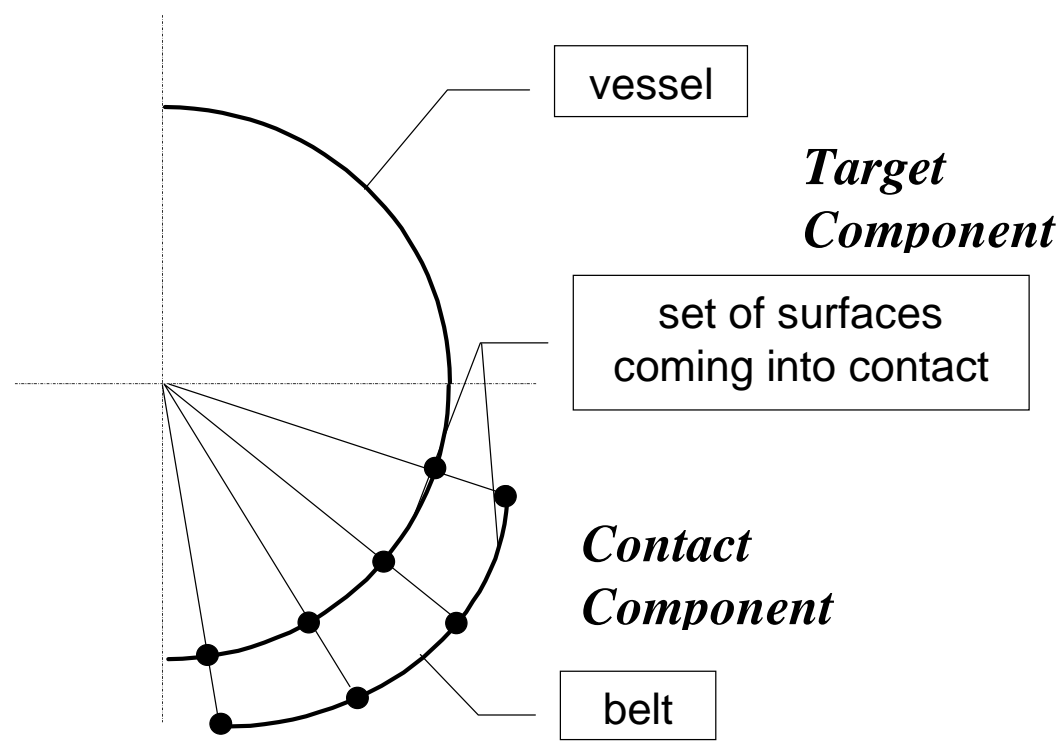

\section{Figure 1. Contact and target surface selection}

Contact occurs when a contact node penetrates the target base within some tolerance. Although elastic Coulomb friction and rigid Coulomb friction are allowed, a frictionless interface was assumed between the vessel and the sling. The interface contact stiffness $K N$, is required as input to the program and can be calculated using the relationship,

$$
K N=c \times E \times h
$$

where $c$ is a factor that controls contact compatibility, usually between 0.01 and $100^{13}, E$ is the smaller Young's modulus when considering contact between two different materials, and $h$ is the characteristic contact length. In 3-D configurations, $h$ should be equal to a typical contact target length, taken here as the square root of the target surface.

In order to achieve convergence and ensure that contact occurs between the two components, one stiff and one flexible, a balance must be struck between the chosen contact stiffness value and the lower Young's modulus. A convergence study was undertaken for a two load step analysis. Firstly, the sling and shell are brought into contact by the use of a fixed 
displacement applied to the top of the sling denoted as LS1. The second load step is the application of gravity to the vessel (LS2). The compatibility factor, $c$, and the belt axial Young's Modulus $E Y \_B$ were varied and the analysis time taken to achieve convergence observed. The criteria chosen to measure the capability of the model to converge were the total number of equilibrium iterations (iter) and the number of bisections (bis) used by the Newton-Raphson algorithm.

\begin{tabular}{|c|c|c|c|c|c|c|c|c|c|c|c|c|}
\hline \multicolumn{13}{|c|}{ Number of iterations and bisections } \\
\hline \multirow{4}{*}{$\begin{array}{c}\begin{array}{c}\text { Values } \\
\text { of }\end{array} \\
E Y \_B \\
\left(\mathbf{N} / \mathbf{m m}^{2}\right)\end{array}$} & \multicolumn{12}{|c|}{ Values of $c^{*}$} \\
\hline & \multicolumn{4}{|c|}{0.01} & \multicolumn{4}{|c|}{0.05} & \multicolumn{4}{|c|}{0.1} \\
\hline & \multicolumn{2}{|c|}{ LS 1} & \multicolumn{2}{|c|}{ LS 2} & \multicolumn{2}{|c|}{ LS 1} & \multicolumn{2}{|c|}{ LS 2} & \multicolumn{2}{|c|}{ LS 1} & \multicolumn{2}{|c|}{ LS 2} \\
\hline & bis. & iter & bis. & iter. & bis. & iter & bis. & iter. & bis. & iter & bis. & iter. \\
\hline 25,000 & 0 & 10 & 1 & 21 & 0 & 16 & 12 & 46 & - & - & - & - \\
\hline 20,000 & 0 & 10 & 3 & 21 & 0 & 16 & - & - & - & - & - & - \\
\hline 15,000 & 0 & 10 & 0 & 16 & 0 & 19 & - & - & - & - & - & - \\
\hline 10,000 & 0 & 10 & 6 & 27 & 0 & 14 & - & - & - & - & - & - \\
\hline 5,000 & 0 & 10 & 1 & 18 & 0 & 15 & - & - & - & - & - & - \\
\hline
\end{tabular}

*For $0.1<\mathrm{c}<100$ failed to achieve convergent solutions

\section{Table 1: Convergence test results for composite SHELL91 model}

From Table 1, it appears that the belt axial Young's modulus $E Y \_B$ does not have much influence on analysis convergence. Although the third series of tests run with composite shell element, SHELL91, tends to show the contrary revealing convergence failure for values under $25,000 \mathrm{~N} / \mathrm{mm}^{2}$ at $c=0.1$. Concerning the compatibility factor, evidence is that the higher the value, the longer it took to achieve convergence. From $c=0.1$ to $\mathrm{c}=100$, no convergence was obtained in any of the cases. Therefore, from the convergence study, the following values of the contact parameters were selected for the purposes of carrying out a geometric parameter study.

- $\boldsymbol{c}=\mathbf{0 . 0 1}$. This value showed the best results and in addition matched with the requirement of a smooth contact between the vessel and the sling, i.e. a small value for the contact stiffness, $K N$.

- $\boldsymbol{E} \boldsymbol{Y}_{-} \boldsymbol{B}=\mathbf{5 , 0 0 0} \mathbf{N} / \mathrm{mm}^{2}$, athough the results of the variation in belt axial stiffness were not significant.

\section{Geometric Parameter Study}

A fully-parametric finite element model of an orthotropic shell and flexible sling support was developed to allow a wide range of geometric parameters to be varied. As a result, a large number of parameters were involved in the model generation. In particular special attention was paid to the parameterisation of the vessel 'sling zone' where contact occurs between the vessel 
and the belt and its neighbouring zone or 'study zone', where maximum strain levels were anticipated to occur.

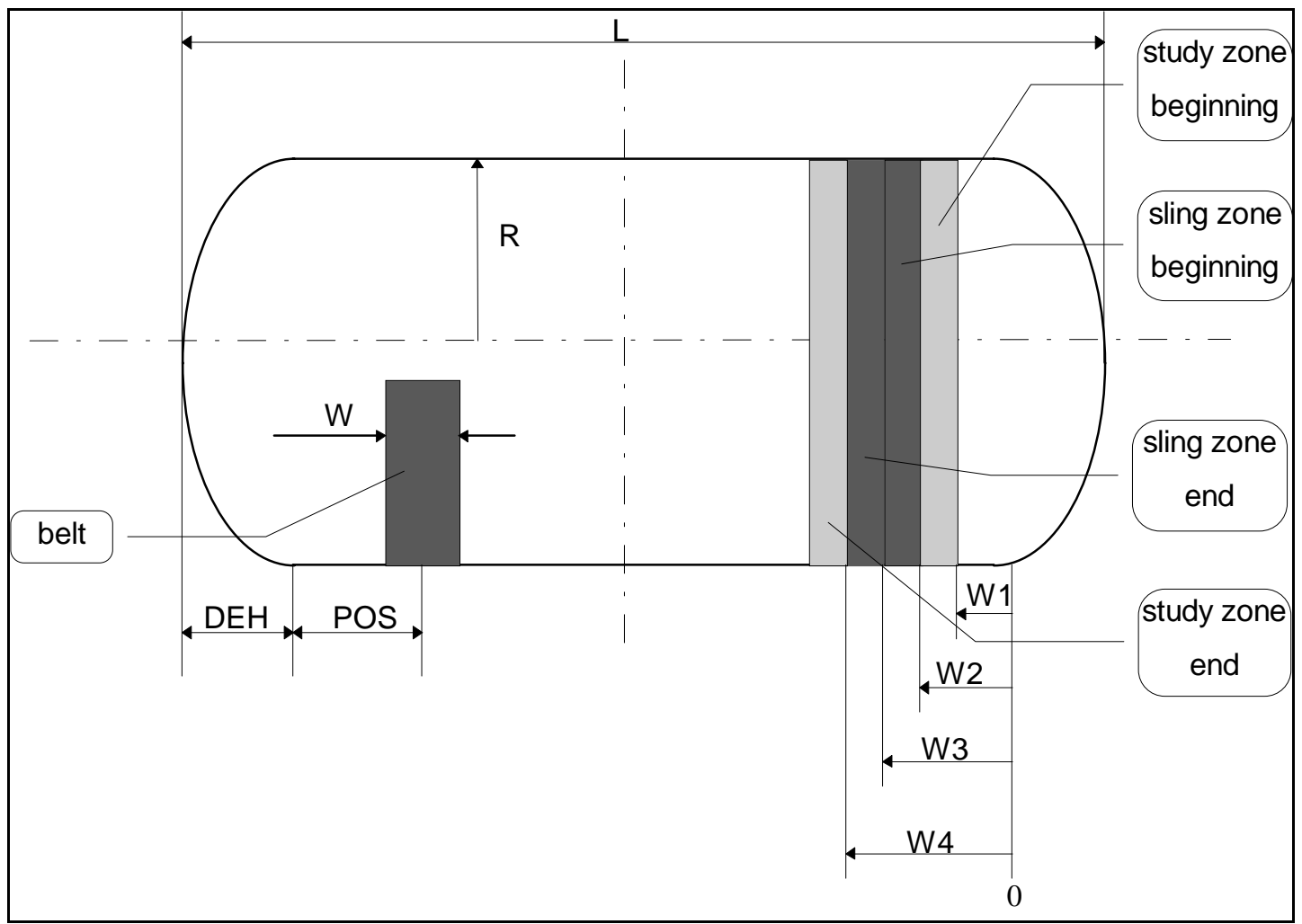

Figure 2. Main finite element parameters

The following data were used to define both the vessel and the belt models. For the vessel, two sets of data are provided referring to an isotropic and an orthotropic approach to the problem. Indeed, given the difficulty of obtaining a converging orthotropic model, the isotropic structure was initially employed to help understand and implement the analysis procedure. Details of the isotropic results can be found in Reference 16. The information obtained allowed the generation of a correct orthotropic model, which was then compared to the isotropic one. All dimensions presented are in millimetres and the reference names in parenthesis designate the parameters used in the ANSYS program (see Figure 2.)

\begin{tabular}{|c|c|c|c|}
\hline Isotropic vessel & \multicolumn{2}{|c|}{ Thickness $\left(T_{-} V\right)$} & 10.75 \\
\hline \multirow[t]{3}{*}{ Orthotropic vessel } & \multicolumn{2}{|c|}{ Outer layer thickness (TT_CSM) } & 4.01 \\
\hline & \multicolumn{2}{|c|}{ Middle-surface layer thickness (T_FW) } & 4.57 \\
\hline & \multicolumn{2}{|c|}{ Inner layer thickness (TB_CSM) } & 2.15 \\
\hline Belt & \multicolumn{2}{|c|}{ Belt thickness (T_B) } & 2.00 \\
\hline \multicolumn{2}{|l|}{ Vessel half-length (L) } & \multicolumn{2}{|c|}{2,000} \\
\hline \multicolumn{2}{|c|}{ Vessel mean radius $(\mathrm{R})$} & \multicolumn{2}{|c|}{$1016+\left(\mathrm{T}_{\text {tot }} / 2\right)=1021.375$} \\
\hline \multicolumn{2}{|c|}{ Dished end height (DEH) } & \multicolumn{2}{|c|}{393} \\
\hline \multicolumn{2}{|l|}{ Belt width $(\mathrm{W})$} & \multicolumn{2}{|c|}{$200 \& 400$} \\
\hline \multicolumn{2}{|l|}{ Belt position (POS) } & \multicolumn{2}{|c|}{$500,750 \& 1000$} \\
\hline \multicolumn{2}{|c|}{ Study zone beginning (W1) } & \multicolumn{2}{|c|}{ POS- $(1.5 \times W)$} \\
\hline \multicolumn{2}{|c|}{ Sling zone beginning (W2) } & \multicolumn{2}{|c|}{ POS $-(0.5 \times W)$} \\
\hline \multicolumn{2}{|l|}{ Sling zone end (W3) } & \multicolumn{2}{|c|}{ POS $+(0.5 \times W)$} \\
\hline
\end{tabular}




\section{Table 2. Main geometric parameters and their variations}

The wrap-round support angle was varied from $120^{\circ}$ to $180^{\circ}$ with an increment of $20^{\circ}$. One quarter of the structure was modelled due to the presence of two planes of symmetry; thus only half of the angle was necessary varying from $60^{\circ}$ to $90^{\circ}$ with an increment of $10^{\circ}$. The vessel density was assumed to equal $2000 \mathrm{~kg} / \mathrm{m}^{3}$. The following properties correspond to those of the orthotropic test vessel.

\begin{tabular}{|l|c|}
\hline CSM layers (outer and inner) - isotropic & $6,160 \mathrm{~N} / \mathrm{mm}^{2}$ \\
\hline Young's modulus (E_CSM) & 0.32 \\
\hline Poisson's ratios (NU_CSM) & $2,330 \mathrm{~N} / \mathrm{mm}^{2}$ \\
\hline Shear modulus (G_CSM) & \\
\hline \multicolumn{2}{|c|}{} \\
\hline FW layer (middle) - orthotropic & $8,270 \mathrm{~N} / \mathrm{mm}^{2}$ \\
\hline Axial Young's modulus (EX_FW) & $38,600 \mathrm{~N} / \mathrm{mm}^{2}$ \\
\hline Circumferential Young's modulus (EY_FW) & 0.26 \\
\hline Major Poisson's ratios (PRXY_FW) & 0.0557 \\
\hline Minor Poisson's ratios (PRYZ_FW) & $4,140 \mathrm{~N} / \mathrm{mm}^{2}$ \\
\hline Shear modulus (GXY_V) & \\
\hline
\end{tabular}

The following properties correspond to those for the sling.

\begin{tabular}{|l|c|}
\hline Circumferential Young's modulus (EX_B) & $125,000 \mathrm{~N} / \mathrm{mm}^{2}$ \\
\hline Axial Young's modulus (EY_B) & $5,000 \mathrm{~N} / \mathrm{mm}^{2}$ \\
\hline Major Poisson's ratio (PRXY_B) & 0.3 \\
\hline Minor Poisson's ratio (PRYZ_B) & 0.06 \\
\hline
\end{tabular}

\section{Tables $3 a$ \& $b$. Material properties for vessel and sling}

The vessel was subjected to a three stage loading history. Since the shell and sling are modelled as discrete components a fixed distance apart, the first load step effectively closes the gap and initiates contact. A second load step introduces the gravitational acceleration vector. This is followed by the third and final load step applying the hydrostatic fluid load in ten equal increments as used in previous experimental work ${ }^{14}$.

\section{Results and Comparisons}

The FE results closely match the experimental patterns recorded by Stafford ${ }^{15}$. Maximum strains were consistently found near the extremities of the supports and located in a circumferential orientation irrespective of lay-up and configuration. A typical strain distribution is presented in Figure 3 for a support angle of $180^{\circ}$. 


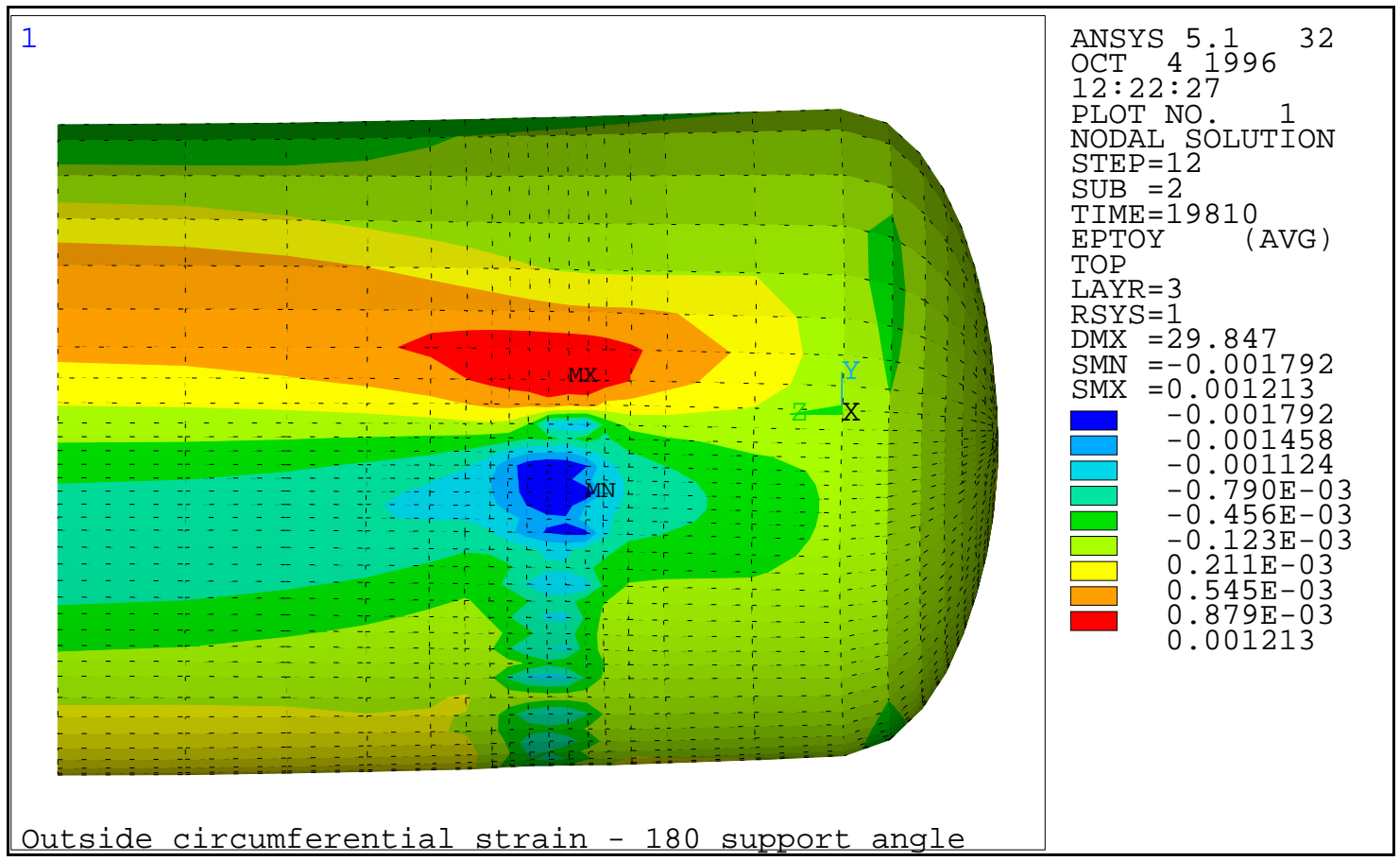

Units are in strain: Maximum compressive value shown is $1792 \mu \varepsilon$; maximum tensile is $1213 \mu \varepsilon$

Figure 3. Outside circumferential strain distribution for $180^{\circ}$ sling for orthotropic vessel

With regard to circumferential strains at the point where the sling parts from the vessel the strain was found to be substantially lower than the saddle support case where the horn strain is maximum. However, the maximum outside compressive strain has moved to a new location some $10^{\circ}$ to $20^{\circ}$ before the 'horn'. This strain then reverses to a maximum tensile strain again at a distance between $10^{\circ}$ and $20^{\circ}$ after the belt loses contact with the vessel, which correlates with experimental results. Also circumferential strains on the inside of the vessel were significantly lower than on the outside (see Table 5 for details of strain values). In addition, strains obtained at the vessel middle-span were also much less than those at the support. These results augur well for the sling-supported vessel. In particular, they indicate the absence of large bending strains, which are damaging at the horn of the conventional saddlesupported vessel.

The following factors are worthy of particular note:

1. The tensile strains are well below the damaging value of $2000 \mu \varepsilon$.

2. The maximum tensile strain occurs on the outside of the vessel. This is of significance when corrosive liquid is being stored. In the limit, some damage could be sustainable on the outside. The problem with strains exceeding the design limitation internally is the possibility of ingress of hazardous material into the glass fibre reinforcement thus initiating stress corrosion cracking. For the $180^{\circ}$ support angle, the maximum inner tensile strain is $626 \mu \varepsilon$ thus giving a fair margin of safety. 
3. The compressive values are hovering near the $2000 \mu \varepsilon$ level. However, a value slightly in excess of this should not be considered a major problem, especially when severe strain gradients are not involved. The possibility of micro-buckling has, however, always to be borne in mind.

The above results gave confidence in the method and the following parameter study was thus initiated.

\section{Parameter Study Results}

The following table describes the variation in geometric parameters shown in Table 2, and associates a reference code which was used when undertaking the orthotropic analysis model defined earlier.

\begin{tabular}{|c|c|c|c|c|}
\hline & $\begin{array}{c}\text { Support angle } \\
(2 \times \text { ALPHA })\end{array}$ & $\begin{array}{c}\text { Belt location } \\
\text { (POS) }\end{array}$ & $\begin{array}{c}\text { Belt width } \\
\text { (W) }\end{array}$ & $\begin{array}{c}\text { Mesh } \\
\text { density }\end{array}$ \\
\hline Ref-dense & $180^{\circ}$ & & & dense \\
\hline Ang-80 & $160^{\circ}$ & \multirow[t]{3}{*}{750} & \multirow{5}{*}{200} & \multirow{6}{*}{ dense } \\
\hline Ang-70 & $140^{\circ}$ & & & \\
\hline Ang-60 & $120^{\circ}$ & & & \\
\hline Pos-1000 & \multirow{3}{*}{$180^{\circ}$} & 1000 & & \\
\hline Pos-500 & & 500 & & \\
\hline Width-400 & & 750 & 400 & \\
\hline
\end{tabular}

Table 4. Reference codes

\begin{tabular}{|l|c|c|c|c|}
\cline { 2 - 5 } \multicolumn{1}{c|}{} & \multicolumn{2}{c|}{$\begin{array}{c}\text { Maximum } \\
\text { compressive strain }\end{array}$} & \multicolumn{2}{c|}{$\begin{array}{c}\text { Maximum } \\
\text { tensile strain }\end{array}$} \\
\hline & Outer & Inner & Outer & Inner \\
\hline Ref-dense & 1,792 & 924 & 1,213 & 626 \\
\hline Ang-80 & 2,445 & 929 & 1,293 & 680 \\
\hline Ang-70 & 2,153 & 944 & 1,316 & 728 \\
\hline Ang-60 & 2,255 & 1,023 & 1,432 & 792 \\
\hline Pos-1000 & 2,009 & 913 & 1,292 & 704 \\
\hline Pos-500 & 1,907 & 889 & 1,075 & 555 \\
\hline Width-400 & 1,371 & 738 & 1,032 & 511 \\
\hline
\end{tabular}

*Note: Locations of these maximums vary - see text and appendix

Table 5. Maximum circumferential strains for orthotropic models $(\mu \varepsilon)$

\begin{tabular}{|l|c|c|c|}
\cline { 2 - 4 } \multicolumn{1}{c|}{} & $\begin{array}{c}\text { Zenith displacement } \\
\text { at central profile }\end{array}$ & $\begin{array}{c}\text { Nadir displacement } \\
\text { at central profile }\end{array}$ & Difference \\
\hline Ref-dense & 26.4 & 29.8 & 3.4 \\
\hline Ang-80 & 21.8 & 33.1 & 11.3 \\
\hline Ang-70 & 17.4 & 31.2 & 13.8 \\
\hline Ang-60 & 16.7 & 34.4 & 17.7 \\
\hline Pos-1000 & 30.4 & 33.7 & 3.3 \\
\hline Pos-500 & 21.6 & 25.0 & 3.4 \\
\hline
\end{tabular}




\begin{tabular}{|l|l|l|l|}
\hline Width-400 & 23.3 & 24.6 & 1.3 \\
\hline
\end{tabular}

\section{Table 6. Maximum displacements for orthotropic models (mm)}

In general terms it is noted, from Table 5, that the overall data recorded for circumferential compressive strains are very close to the design limit of 2,000 $\mu \varepsilon$ given by BS 4994 and even exceeded it in the $120^{\circ}, 140^{\circ}$ and $160^{\circ}$ angle support cases. However, it should be remembered (as indicated above) that the limit is based on a tensile strain consideration. Compressive strain values are generally permitted to go a little higher. Strain plots are shown in Appendix 1. These describe the variation in outside circumferential strain with varying geometric parameters and also indicate the locations for the maximum compressive and maximum tensile strain, denoted $\mathrm{MN}$ and $\mathrm{MX}$ respectively on the plots.

Concerning the displacements, Table 6 gives details of the displacements at the central profile and Figure 4 presents three different views of the vessel deformed shape. The overall sagging of the vessel at its centre can easily be seen on the front view. In the bottom view, the effect of the sling can also be noted. It creates a slight inflexion on the vessel generating tensile strains. The section view reveals interesting information about the belt action on the vessel and its consequences. It clearly shows that the vessel constrained by its sling support can only expand above the horn. This explains compressive strains where contact exists and tensile strains where the belt parts from the vessel, giving a virtually zero strain transition zone at the horn.

Considering the variation in geometric parameters, the following comments are made. With respect to support angle, the experimental data did not show significant variation in results for different support angle. The FEA results however show a slight increase in the maximum strain as the support angle was reduced. Nevertheless these strain variations remain far less than those recorded with a saddle arrangement at the same point. 


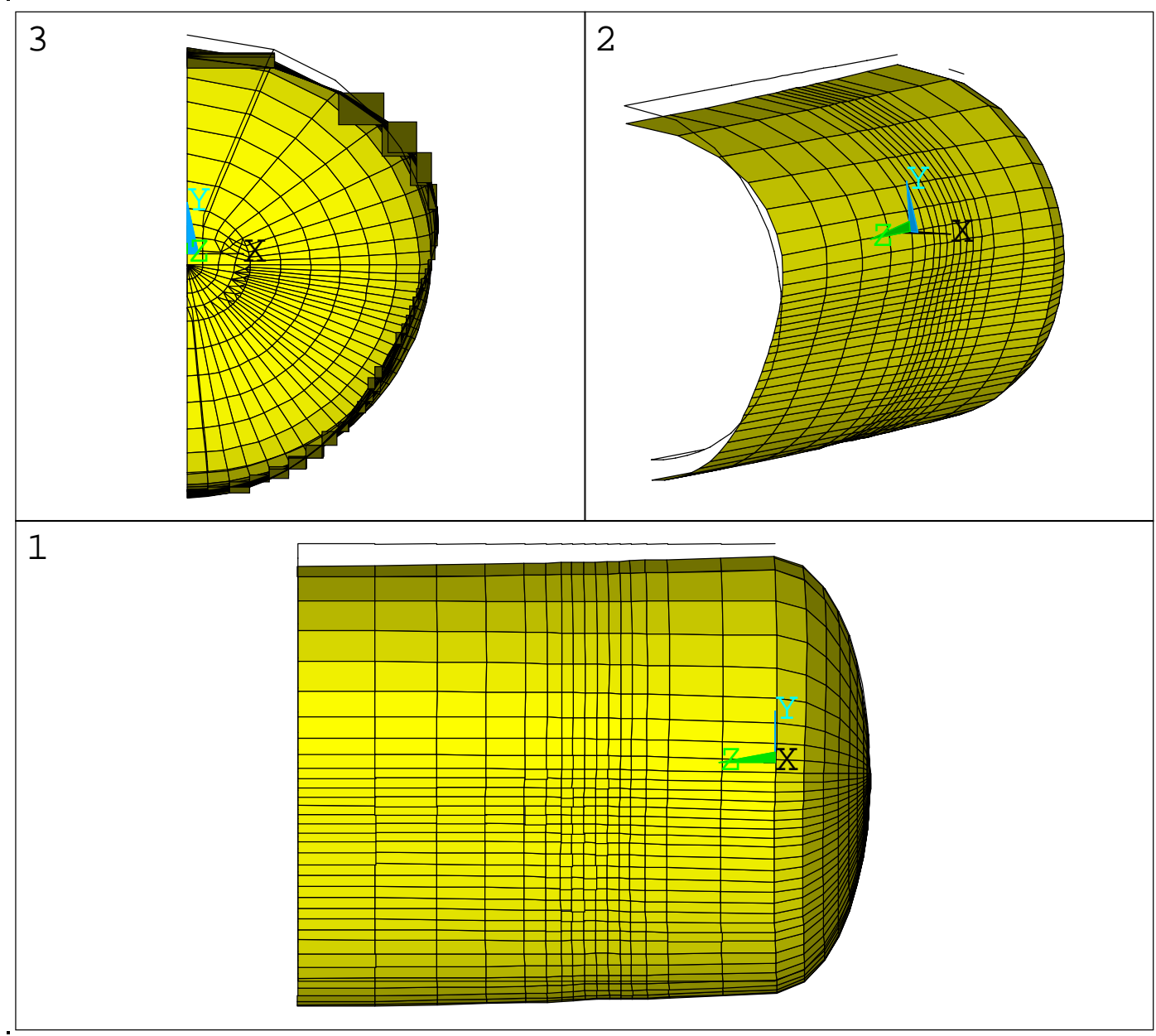

\section{Figure 4. Typical vessel deformed shape}

Moving the sling from its initial position, i.e. $750 \mathrm{~mm}$ from the edge of the vessel cylindrical shell, did not result in major differences in any of the strains. In general, a variation between $-100 \mu \varepsilon$ and $+200 \mu \varepsilon$ for the strains relative to the $750 \mathrm{~mm}$ location was observed; this with the same maximum displacement at the central profile. The strain difference may be due in part to the compact dimensions of the vessel under examination (length: $4 \mathrm{~m}$ - diameter: $2 \mathrm{~m}$ ) which minimises bending effects on the vessel shell itself and thus the significance of the sling location. However, the results show that the closer the belt is to the vessel ends, the lower the strains and displacements, which is certainly due to the natural stiffness of the vessel ends.

The variation in sling width from twice the original dimension showed interesting reductions both on strain and displacement figures. In the circumferential direction, compressive strains (the larger values) were reduced by about $\mathbf{2 5 \%}$ and to a lesser extent tensile strains by around $15 \%$. In the axial direction, a significant reduction was obtained with values $12 \%$ lower (peak value at 27\%) for compressive strains and between 5 and $10 \%$ for the tensile strains. From a displacement point of view, results were again encouraging, with a reduction of $62 \%$ in maximum deflection at the central profile. 


\section{Conclusions}

Comparisons between the present analysis and published experimental results ${ }^{14,15}$ show reasonable agreement. However, the finite element models produce results consistently higher than those measured experimentally. This points towards a useful design tool. Examination of the deformed shapes of the models indicates the effectiveness of the system in offering a smooth contact interface. The model also presented an insight into the sensitivity of the system to sling stiffness and constraint condition.

The parameter study showed some behavioural similarities to the conventional saddle support. Large wrap round angles proved slightly beneficial and locating the sling near the end of the vessel ensured additional support was achieved; this resulting in lower strain levels. Increasing the width of the sling showed the greatest benefit with, in some cases, a $25 \%$ reduction resulting.

Perhaps the most significant conclusion is the lack of large bending effects, which are normally present in the case of the saddle support vessel (the most common method of support). In addition, the tensile strains are significantly lower than the compressive values. Since the tensile strains are the most damaging, this leads to promising possibilities.

While further work on more general cases is still required, the present treatment indicates potential for the sling-supported method.

\footnotetext{
${ }^{1}$ Zick, L. P. 'Stresses in large horizontal cylindrical vessels on two saddle supports.' Weld. Res. J. Suppl., 30, (1951) 435-S-45-S

${ }^{2}$ BS 4994, 'Specification for the Design and construction of vessels and tanks in reinforced plastics', British Standards Institution, London, 1987

${ }^{3}$ ASME Boiler and Pressure Vessel Code, Section X, 'Fiberglass-reinforced plastic pressure vessels' Appendices 1-5, Attachments and supports, 1995

${ }^{4}$ BS 5500, 'Specification for Unfired fusion welded pressure vessels', British Standards Institution, London, 1997

${ }^{5}$ Warrender, A.J., Wood, J., Tooth, A.S. \& Banks, W.M. 'The support of horizontal cylindrical GRP vessels saddles or longitudinal beams?' $4^{\text {th }}$ International Conference on Composite Structures, Paisley, July 1987 (Ed. I H Marshall), Vol 1, Analysis and design studies, 1987, Ch 17, pp 1.235-1.252, Elsevier Applied Science.

${ }^{6}$ Banks, W.M., Tooth, A.S. \& Ong, L.S. 'The support of horizontal multi-layered GRP vessels: Saddles, slings or longitudinal beams?' $8^{\text {th }}$ International Conference Pressure Vessel Technology, American Society of Engineers, Montreal, Canada,1996, Vol. 2, pp. 315-322

${ }^{7}$ Duthie, G. \& Tooth, A. S. 'The analysis of horizontal cylindrical vessels supported by saddles welded to the vessel - a comparison of theory and experiment' $3^{\text {rd }}$ International Conference Pressure Vessel Technology,. American Society of Engineers, New York, USA, 1977, pp. 25-38

${ }^{8}$ Tooth, A. S., Duthie, G., White, G. C. \& Carmichael, J., 'Stresses in horizontal storage vessels - a comparison of theory and experiment.' J. Strain Anal., 17 (1982) 169-76.

${ }^{9}$ Tooth, A. S.\& Nash, D. H., 'Stress analysis and fatigue assessment of twin saddle supported pressure vessels.' Pressure Vessels and Components, ASME Conf. American Society of Mechanical Engineers, New York, USA, 1991, pp 41-8.

${ }^{10}$ Tooth, A.S., Banks, W.M., Seah, C.P., \& Tolson, B.A., ' The twin-saddle support of horizontal multi-layered GRP vessels - theoretical analysis, experimental work and a design approach' Proc. Instn. Mech. Engrs. Vol. 208 pp59-74, I MechE, London, 1994

${ }^{11}$ Erzingatzian, A., Hoa, S.V. \& Xiao, X., 'Mechanical behaviour of filament wound FRP pipes on saddles supports.' $8^{\text {th }}$ International Conference on Pressure Vessel Technology, American Society of Engineers, Montreal, Canada,1996, pp 123-123

${ }^{12}$ Nash, D.H., Banks, W.M., \& Bernaudon, F., 'Finite element modelling of sling supported vessels', Bi-Centenary Conference on Thin Walled Structures, University of Strathclyde, Glasgow, December 1996
} 
${ }^{13}$ ANSYS Structural Analysis Guide, Chapter 8, Non-linear Structural Analysis, $1^{\text {st }}$ Edition, SAS IP, 1996

${ }^{14}$ Guyon, B., 'Experimental study of the belt support of horizontal cylindrical GRP vessels' Undergraduate Thesis, University of Strathclyde, 1993

${ }^{15}$ Stafford, G., 'Support and local load problems associated with GRP vessels.' Undergraduate Thesis, University of Strathclyde, 1994

${ }^{16}$ Bernaudon, F., 'The study of belt supported glass reinforced plastic pressure vessels using finite element analysis.' ' Undergraduate Thesis, University of Strathclyde, 1996 